\title{
Atypical Presentation of Charles Bonnet Syndrome: Frightening Hallucinations Coinciding with
}

\section{Hypertensive Urgency}

\section{Amanda M. Warren ${ }^{1 *}$, Dana Lin ${ }^{1}$}

${ }^{1}$ Department of Family Medicine, St. George's University, The Brooklyn Hospital Center, True Blue, Grenada, United States.

* Corresponding Author: Amanda M. Warren, ${ }^{1}$ Department of Family Medicine, St. George's University, The Brooklyn Hospital Center, True Blue, Grenada, United States.

Received date: 12 February 2021; Accepted date: 19 February 2021; Published date: 23 February 2021

Citation: Warren AM, Lin D. Atypical Presentation of Charles Bonnet Syndrome: Frightening Hallucinations Coinciding with Hypertensive Urgency. J Med Case Rep Case Series 2(4): https://doi.org/10.38207/jmcrcs20210007

Copyright: (C) 2021 Warren AM. This is an open-access article distributed under the terms of the Creative Commons Attribution License, which permits unrestricted use, distribution, and reproduction in any medium, provided the original author and source are credited.

\begin{abstract}
Charles Bonnet syndrome is a condition characterized by the presence of purely visual hallucinations in patients with a history of visual pathway damage and preserved cognitive status. CBS is often overlooked as an etiology for visual hallucinations in the visually impaired, especially in elderly populations, where psychological origins are often first considered. This case report intends to increase awareness of the syndrome, as well as to document an atypical presentation that coincides with hypertensive urgency and malicious imagery. The lack of awareness of this syndrome can lead to an inaccurate diagnosis in a variety of healthcare settings including but not limited to, ophthalmology, psychiatry, neurology, the emergency department, or family medicine practices. Increased understanding of this syndrome can benefit both the physician's approach to diagnosis and patient management.
\end{abstract}

Keywords: charles bonnet syndrome, visual hallucinations, vision loss

\section{Introduction}

Charles Bonnet syndrome is an under-recognized phenomenon amongst patients who experience vision loss and may be more common than is currently reported. CBS, also known as visual release hallucinations, occurs in approximately 10-38\% of patients with decreased visual function. These visual perceptions tend to be benign in nature, frequently featuring people, animals, or inanimate objects [4]. This most commonly affects the geriatric population, reflective of the increased prevalence of blindness amongst older patients. The wide range in prevalence is likely due to the under-reporting by

\section{Case Report}

An 83-year-old African American female with a past medical history of hypertension, type 2 diabetes, chronic kidney disease, asthma, left eye cataracts, and temporal arteritis leading to a 12-year history of bilateral eye blindness presented to the ED with complaints of unsteady gait and visual hallucinations. The patient described an increased frequency of episodic and binocular complex visual hallucinations eight days prior to admission. While experiencing the hallucinations the patient maintained full insight and awareness of the fictional nature of her experience and stated cessation of the hallucinations was often prompted by confirmation that what she was experiencing was in fact illusory. The first hallucination the patient had was of cats and dogs several years after she became blind. The following hallucination was almost a decade later, approximately two patients in fear of being labeled with a mental illness, as well as underrecognition of the condition by physicians. Some patients report the ability to make them disappear simply by blinking repeatedly or looking directly at the subject. An interesting feature of this syndrome is that most patients are aware after their first hallucination that these images are fictitious. Reassurance that these hallucinations are a natural consequence of vision loss can reduce the frequency of occurrence.

weeks before presenting to the ED featuring three female children. Just a few days after, the following hallucination featured a single helicopter and several airplanes. The patient stated her hallucinations most commonly initiate outdoors and normally last a few minutes. Upon physical examination, the patient was noted to have a blood pressure of $213 / 87$. The patient has subsequently treated with Nifedipine $90 \mathrm{mg}$ and reported cessation of the visual hallucinations. The patient was admitted to ED again 4 days after her initial admission, with similar complaints of unsteady gait. After evaluation, she was noted to have another episode of hypertensive urgency with a blood pressure of 190/80, also treated with Nifedipine $90 \mathrm{mg}$. The patient stated that prior to her second admission her visual hallucination consisted of seeing boxes of bugs around her apartment, 
and bugs crawling on her lower extremities bilaterally. The patient denied the presence of any auditory, tactile, or olfactory hallucinations during this incident. She also reported having a hard time accepting the fictional nature of what she was experiencing despite repeated denials of its manifestation by her neighbor. This hallucination was unusually long compared to previous hallucinations, lasting 1.5 hours before resolving. On physical

\section{Discussion}

In our case, the patient was admitted, not because of the hallucinations, but due to two consecutive cases of hypertensive urgency. It was only after a thorough evaluation that the patient reported not only the presence, but also an increased frequency of visual hallucinations prior to admission. It is possible that hypertensive urgency affects the brain via two pathways later described, as both times the patient presented with hypertensive urgency, there was an associated hallucination. It has been suggested that a hypertensive crisis could potentially cause a series of functional changes to the differentiated cortical areas, which could act as a catalyst, triggering visual hallucinations. There has also been another documented case of Charles Bonnet Syndrome occurring secondary to hypertensive crisis, with frequency of hallucinations progressively reducing as arterial pressure diminished [1]. Patients with Charles Bonnet syndrome more frequently present with simple hallucinations consisting of lights, colors, or elementary shapes. Complex hallucinations are not uncommon amongst this patient population, and typically present as neutral subjects such as people, animals, and objects. One notable aspect of the patient's most recent hallucination was its atypical presentation: She visualized bugs crawling everywhere which frightened her, whereas typical visual release hallucinations are benign and do not commonly cause direct distress in patients. Normally those with the condition are also aware that the visions they see are not real, however during her most recent episode, the patient had difficulty being convinced of the fictional nature of her hallucinations $[\mathbf{2 , 3}, \mathbf{4}]$.

The patient also reported previous episodes to last for a few minutes, examination, during both encounters, the patient was awake, alert, and oriented. The patient's score for MMSE adjusted for vision impairment was 27.8 , ruling out possible dementia or delirium. Once the patient's blood pressure was stabilized, she was informed about her diagnosis of Charles Bonnet Syndrome, which subsequently eased her concern about the unexplained hallucinations.

whereas this hallucination was present for over an hour. The increased sympathetic stimulation due to the patient's distress secondary to the visual hallucinations or the patient's refusal to believe the fictional nature of her hallucination may have played a role in the prolonged duration of the episode. Although the mechanism behind the visual hallucinations associated with Charles Bonnet syndrome are not universally definitive, several theories have been proposed. The Release Phenomenon embodies the juxtaposition between "phantom sensations" as seen in amputees that present with tactile sensations in their missing limb; this syndrome the phantom sensation presents as visual hallucinations, embodying the affected sensory domain. Pathological interference within the visual pathway disrupts afferent sensory input to the brain thereby increasing activity in the central nervous system, which the body misinterprets as hallucinations. This proposed mechanism of abnormal visual processing is why Charles Bonnet syndrome is sometimes referred to as "phantom vision" $[2,7,8]$. Another proposed theory is the Deafferentation Theory, which describes the idea that loss of visual input to the brain will result in a change in the excitability of the visual association cortex. This theory is also associated with ocular pathology or damage to visual pathways but asserts that the damage leads to spontaneous neuronal discharge to occur causing increased excitability within the visual association cortex, thus producing the visual hallucinations. Due to the plasticity of the visual system in conjunction with a lower threshold for activation in the neurons, stimulus may be able to propagate along new axons in a reorganized receptive field $[\mathbf{5 , 6 , 7 , 8 ]}$.

Table 1: Diagnostic Criteria for Charles Bonnet Syndrome

\begin{tabular}{|l|}
\hline 1. At least one visual hallucination \\
\hline 2. A period between the first and last hallucination exceeding 4 weeks \\
\hline 3. Full or partial retention of insight into the unreal nature of thehallucinations \\
\hline 4. Absence of hallucinations in other sensory modalities \\
\hline 5. Absence of delusions \\
\hline
\end{tabular}

Due to the lack of discussion around Charles Bonnet Syndrome, it is often overlooked as an etiology for benign visual hallucinations. This is not considered a psychiatric related disorder due to the patient's insight into the fictional etiology of their visual experiencesand lack of hallucinations present in other sensory modalities [9]. CBS commonly presents in elderly populations with a mean age of 70-85 
years and affected patients typically have a history of diminished visual acuity or visual field loss. The presence of these visual release hallucinations is frequently under-reported by patients in fear of judgment and stigma against mental health disorders [10]. Approximately $60 \%$ of patients surveyed with Charles Bonnet Syndrome are wary of telling their physicians about their hallucinations in fear of being diagnosed with a mental illness [3]. An additional concern arises as the condition is simultaneously underrecognized by clinicians. A study conducted amongst family physicians in Canada determined over half the doctors were completely unaware of the Charles Bonnet Syndrome. Of the physicians who were, nearly three-quarters of them stated they had at least one patient annually who are afflicted [3]. Charles Bonnet syndrome is often misattributed to a psychological condition or can become costly to diagnose with extensive imaging done by physicians simply unaware of the syndrome. This misdiagnosis not only adds stress to the patients but also can elicit unnecessary testing and

\section{Conclusion}

Although Charles Bonnet syndrome is a diagnosis of exclusion, recognizing the symptoms indicated in (Table 1) can effectively ensure diagnosis and management of patients with this visual release syndrome. Recognition of the disorder is essential as reassurance from the physician plays a crucial role in pacifying patient concerns and aiding in management. Misdiagnosis can cause distress to the consults from a variety of specialties. A study performed in 2008, noted that $51.5 \%$ of participants reported mild stress compared to 30 $\%$ of participants who reported severe stress secondary to the presence of the visual hallucinations. Participants of this study surprisingly conveyed that the stress they reported was not a direct reaction to the quality of the images they were experiencing, but the stress was primarily due to concern about the underlying etiology of the hallucinations [7,9]. This further elucidates the importance of accurate diagnosis in patients with this syndrome. No wellestablished medications are currently available to treat this condition. Some incidences of severe Charles Bonnet Syndrome have been treated with antipsychotics but are generally not used on patients with purely benign visual hallucinations due to the risk of adverse effects and unverified benefit. Management is centered on educating the patients and their families about the benign etiology of their condition and providing them with reassurance, which can improve the patient's status without pharmacological intervention.

patient as well as elicit unnecessary costs due to consults and additional testing. Awareness of the disorder may potentially increase prevalence as patients may come forward with testimonies of similar cases; comfortable with exposing their hallucinations after understanding it is a normal consequence of their vision loss.

Conflict of Interest: The authors declare they have no competing interests.

\section{References}

1. Cifuentes-Canorea P, Cerván-López I, Rodríguez-Uña I, SantosBueso E (2018) Síndrome de Charles Bonnet secundario a crisis hipertensiva. Neurología. 33(7): 473-474.

2. Cogan DG (1973) Visual hallucinations as release phenomena. Graefes Arch Clin Exp Opthalmol.188(2): 139-150.

3. Gordon KD, Felfeli T (2018) Family physician awareness of Charles Bonnet Syndrome. Fam Pract. 18;35(5): 595-598.

4. Jackson ML, Ferencz J (2009) Charles Bonnet Syndrome: visual loss and hallucinations. CMAJ. 181(3-4): 175-176.

5. Kazui H, Ishii R, Yoshida T, Ikezawa K, Takaya M, et al. (2009) Neuroimaging studies in patients with Charles Bonnet Syndrome. Psychogeriatrics 9(2): 77-84.

6. Kester EM (2009) Charles Bonnet Syndrome: case presentation and literature review. Optometry 80(7): 360-366

7. Pang L (2016) Hallucinations experienced by visually impaired: Charles Bonnet syndrome. Optom Vis Sci. 93(12): 1466-1478.

8. Vale TC, Fernandes LC, Caramelli P (2014) Charles Bonnet syndrome: characteristics of its visual hallucinations and differential diagnosis. Arq Neuropsiquiatr 72(5): 333-336.

9. Vukicevic M, Fitzmaurice K (2008) Butterflies and black lacy patterns: the prevalence and characteristics of Charles Bonnet hallucinations in an Australian population. Clin Experiment Opthalmol 36(7): 659-665.

10. Yacoub R, Ferrucci S (2011) Charles Bonnet syndrome. Optometry 82(7): 421-427. 